\title{
Automatic Identification of Modulation Signals Based On High Order Cumulants
}

\author{
Yanhua Liu ${ }^{1, *}$, Xiaoyan Zhang ${ }^{1, a}$, Haixin Sun ${ }^{1}$, Yuting Yuan ${ }^{1}$, Jianquan Yan $^{1}$ \\ ${ }^{1}$ College of information science and technology, Xiamen University, Xiamen, China \\ * corresponding author: liuyh@xum.edu.cn \\ *liuyh@xum.edu.cn, ${ }^{\mathrm{a}} 15280630552 @ 163 . c o m$
}

Keywords: Automatic modulation classification; cumulants; high order statistics.

\begin{abstract}
The automatic modulation identification for communication signals is an important problem in the intercepted signal processing and widely used in non-cooperation communication of military and commercial. This paper proposes a method that based on the second and fourth order cumulants of received signal which can classify digital modulation signal, which can suppress the Gaussian white noise, so we can identify six digital modulation signals: 2ASK、2PSK、4PSK、 2FSK、4FSK and 16QAM. In the end, the computer simulation that the automatic identification of modulation signals based on high order cumulates is reasonable.
\end{abstract}

\section{Introduction}

Automatic modulation classification (AMC) is used for identification of transmitted signal's constellation by observing received data samples, usually corrupted by noise and multipath fading [1]. Because of their simplicity, pattern-recognition methods of AMC, based on extraction of key features of received signal and determination of modulation technique of the transmitted signal according to pre-designed decision rule, are very popular. As key features in pattern-recognition, higher-order statistics -cumulants and moments, are usually considered.

Because Gaussian white noise has no influence on received signal as the higher order cumulants more than the second order cumulants, the method of higher order cumulants has a good inhibitory effect on noise. At present, the higher order cumulates is widely used in achieving the identification of digital modulation signals. A hierarchical framework based on fourth-order cumulants was proposed in [2]. A combination of second- and fourth-order cyclic cumulants' (CC) magnitudes was proposed for QPSK and QAM signal classification was studied in [3]. A method based on higher order correlations of classifying M-ray frequency-shift keying (MFSK) signals was studied in [4]. While higher-order up to eighth-order CC was adopted in [5] and nth-order warped CC magnitudes were utilized in [6].

In this paper higher-order CC based features are investigated for automatic recognition of linearly modulated signals in additive white Gaussian noise (AWGN). And it can be used as a simple preliminary classifier and may also be applied within each subclass to determine the exact modulation type if sufficient signal-to-noise ratio (SNR) and sample size are available. Second- and fourth-order are used to identify six digital modulation signals: 2ASK、2PSK、4PSK、2FSK、4FSK and 16QAM.

\section{Classification based on High order statistics}

\section{Definitions}

Let $\gamma=\operatorname{col}\left(\gamma_{1}, \gamma_{2}, \cdots, \gamma_{k}\right)$ and $x=\operatorname{col}\left(x_{1}, x_{2}, \cdots, x_{k}\right)$ where $\left(x_{1}, x_{2}, \cdots, x_{k}\right)$ denote a collection of random variables [6]. The $k$ th-order cumulant of these random variables is defined as the coefficient of $\left(\gamma_{1}, \gamma_{2}, \cdots, \gamma_{k}\right)$ in the Taylor series expansion of the cumulant-generating function.

$K(\gamma)=\ln E\left\{\exp \left(j \gamma^{\prime} x\right)\right\}$ 
And let $x(t)$ be a zero-mean $k$ th-order stationary random process. The $k$ th-order cumulant of the random variables $x(t), x\left(t+\tau_{1}\right), \cdots x\left(t+\tau_{k-1}\right)$, i . e .

$$
C_{k x}\left(\tau_{1}, \tau_{2,} \cdots, \tau_{k}\right)=\operatorname{cum}\left(x(t), x\left(t+\tau_{1}\right), \cdots x\left(t+\tau_{k-1}\right)\right)
$$

Because of stationarity, the $k$ th-order cumulant is only a function of the $k-1$ lags $\tau_{1}, \tau_{2}, \cdots, \tau_{k-1}$. The $\tau_{1}-\tau_{2}-\cdots \tau_{k-1}$ space constitutes the domain of support for $C_{k, x}\left(\tau_{1}, \tau_{2}, \cdots, \tau_{k}\right)$. If $x(t)$ is nonstationary, then the $k$ th-order cumulant depends explicitly on $t$ as well as on $\tau_{1}, \tau_{2}, \cdots, \tau_{k-1}$, and we use the notion $C_{k, x}\left(t: \tau_{1}, \tau_{2}, \cdots, \tau_{k-1}\right)$.

$$
\left.\left.C_{k, x}\left(\tau_{1}, \tau_{2}, \cdots, \tau_{k}\right)=E\left(\left\{x\left(\tau_{1}\right)\right\} \cdots x\left(\tau_{k-1}\right)\right)\right\}-E\left\{g\left(\tau_{1}\right)\right\} \cdots g\left(\tau_{k-1}\right)\right\}
$$

Where $\{g(t)\}$ is a Gaussian random process with the same second-order statistic as $\{x(t)\}$. Cumulants, therefore, not only display the amount of high-order correlation, but also provide a measure of the distance of the random process from Gaussianity. Clearly, if $x(t)$ is Gaussian then the cumulants are all zero; this is not only true for $k=3$ and $k=4$, but for all $k$.

The second-, fourth- order cumulants of zero-mean $x(t)$, are

$$
\begin{aligned}
& C_{2, x}(\tau)=E\{(x(t) x(t+\tau)\} \\
& C_{4, x}(\tau)=E\left\{\left(x(t) x\left(t+\tau_{1}\right) x\left(t+\tau_{2}\right) x\left(t+\tau_{3}\right)\right\}-C_{2, x}\left(\tau_{1}\right) C_{2, x}\left(\tau_{2}-\tau_{3}\right)\right. \\
& -C_{2, x}\left(\tau_{2}\right) C_{2, x}\left(\tau_{3}-\tau_{1}\right)-C_{2, x}\left(\tau_{3}\right) C_{2, x}\left(\tau_{1}-\tau_{2}\right)
\end{aligned}
$$

\section{Analysis of the digital modulation signal}

According to the nature of the digital modulation signal, respectively the variable frequency of digital modulation signal expressed as [7]

MASK:

$S(t)=\sqrt{E} \sum_{n} a_{n} p\left(t-n T_{s}\right) \exp \left(j \theta_{c}\right)+n(t), a_{n} \in\{2 m-1-M . m=1,2, \cdots, M\}$

MPSK:

$S(t)=\sqrt{E} \sum_{n} \exp \left(j \varphi_{n}\right) p\left(t-n T_{s}\right) \exp \left(j \theta_{c}\right)+n(t), \varphi_{n} \in\left\{(m-1) \frac{2 \pi}{M} \cdot m=1,2, \cdots, M\right\}$

MFSK:

$S(t)=\sqrt{E} \sum_{n} \exp \left(j \omega_{n} t\right) p\left(t-n T_{s}\right) \exp \left(j \theta_{c}\right)+n(t), \omega_{n} \in\{(2 m-1-M) \square \omega, m=1,2, \cdots, M\}$

QAM:

$S(t)=\sqrt{E} \sum_{n} \exp \left(j \vartheta_{c}\right)\left[a_{k}+b_{k}\right], a_{k}, b_{k} \in\{(2 m-1-\sqrt{M}), m=1,2, \cdots, \sqrt{M}\}$

The cumulants in (4), (5) and (6) can be estimated from the sample estimates of the corresponding moments. We assume that $S(t)$ is zero-mean; in practice, the mean value of sample signal is removed before cumulant estimation. Sample estimating of the correlations are given by

$$
\begin{aligned}
& \hat{C}_{20}=\frac{1}{N} \sum_{n=1}^{N}(S(t))^{2} \\
& \hat{C}_{21}=\frac{1}{N} \sum_{n=1}^{N}|S(t)|^{2}
\end{aligned}
$$

Where the superscript ${ }^{\wedge}$ denotes a sample average. This leads to the following estimates:

$$
\begin{aligned}
& \hat{C}_{40}=\frac{1}{N} \sum_{n=1}^{N}(S(t))^{4}-3 C_{20}{ }^{2} \\
& \hat{C}_{41}=\frac{1}{N} \sum_{n=1}^{N}(S(t))^{3} S^{*}(t)-3 C_{20} C_{21} \\
& \hat{C}_{42}=\frac{1}{N} \sum_{n=1}^{N}|S(t)|^{4}-\left|C_{20}\right|^{2}-2 C_{21}^{2}
\end{aligned}
$$

According to (11) (15), we can calculate the cumulants of the digital modulation signal: 
Table I The cumulants of the digital modulation signals

\begin{tabular}{|c|c|c|c|c|c|}
\hline types & $\left|C_{20}\right|$ & $\left|C_{21}\right|$ & $\left|C_{40}\right|$ & $\left|C_{41}\right|$ & $\left|C_{42}\right|$ \\
\hline 2ASK & $E^{2}$ & $E$ & $E^{2}$ & $E^{2}$ & $E^{2}$ \\
\hline 2PSK & $E^{2}$ & $E$ & $2 E^{2}$ & $2 E^{2}$ & $2 E^{2}$ \\
\hline 4PSK & 0 & $E$ & $E^{2}$ & 0 & $E^{2}$ \\
\hline 2FSK & 0 & $E$ & 0 & 0 & $E^{2}$ \\
\hline 4FSK & 0 & $E$ & 0 & 0 & $E^{2}$ \\
\hline 16QAM & 0 & $E$ & $6.8 E^{2}$ & 0 & $6.8 E^{2}$ \\
\hline
\end{tabular}

Based on the data of table 1, different parameter combinations can be used to identify the most of the digital modulation signal, except the 2FSK and 4FSK signals. However, the 2FSK signal and 4FSK signal can't be distributed after down frequency conversion. It is proved through the processing of the first derivate on equation(10) that the frequency variable modulation signal can be converted to a modulation signal which contains amplitude information. So, the higher order cumulates can be used to identify the 2FSK signal and 4FSK signal.

Table II The cumulants of the MFSK signals using derivate

\begin{tabular}{|c|c|c|}
\hline types & $\left|C_{21}\right|$ & $\left|C_{42}\right|$ \\
\hline $2 \mathrm{FSK}$ & $E \square \omega^{2}$ & $E \square \omega^{4}$ \\
\hline $4 \mathrm{FSK}$ & $5 E \square \omega^{2}$ & $9 E \square \omega^{4}$ \\
\hline
\end{tabular}

\section{Algorithm steps}

The obtained cumulants of signals theoretical value in table I. Because higher order statistic cannot be directly used in digital recognition because of relating with signal power, only the higher order statistic converts to characteristic parameters after processing which can be used for signal recognition algorithm.

$$
\begin{aligned}
& f \times 1=\left|C_{41}\right| /\left|C_{42}\right| \\
& f \times 2=\left|C_{40}\right| /\left|C_{42}\right| \\
& f \times 3=\left|C_{42}\right| /\left|C_{21}\right|^{2}
\end{aligned}
$$

Step1: The signals can be distinguished into two parts by using the parameter 1:2ASK、2PSK and 4PSK、2FSK、4FSK、16QAM. The values are one and zero which can be seen in figure 2-1.

Step2: The signals can be distinguished into 4PSK、16QAM and 2FSK、4FSK by using the parameter 2.The values are respectively one and zero. The simulation is shown in figure 2-2.

Step3: distinguish between two signals of 2PSK and 2ASK signals by using the parameter 3 . The theoretical values are respectively two and one which can be seen in figure 2-3.

Step4: distinguish between two signals of 4PSK and 16QAM signals by using the parameter 3 which the values are respectively one and six point of eight. The simulation is shown in figure 2-4, the parameter of the signal can be effectively identified if the signal to noise ratio is greater than $10 \mathrm{~dB}$.

Step5: according to the table II, the signals of 2FSK and 4FSK can be distinguished by using the parameter 3.It can be seen from the simulation graph in figure 2-5 that the parameter of the signal can be effectively identified as long as the signal to noise ratio of greater than $15 \mathrm{~dB}$. 

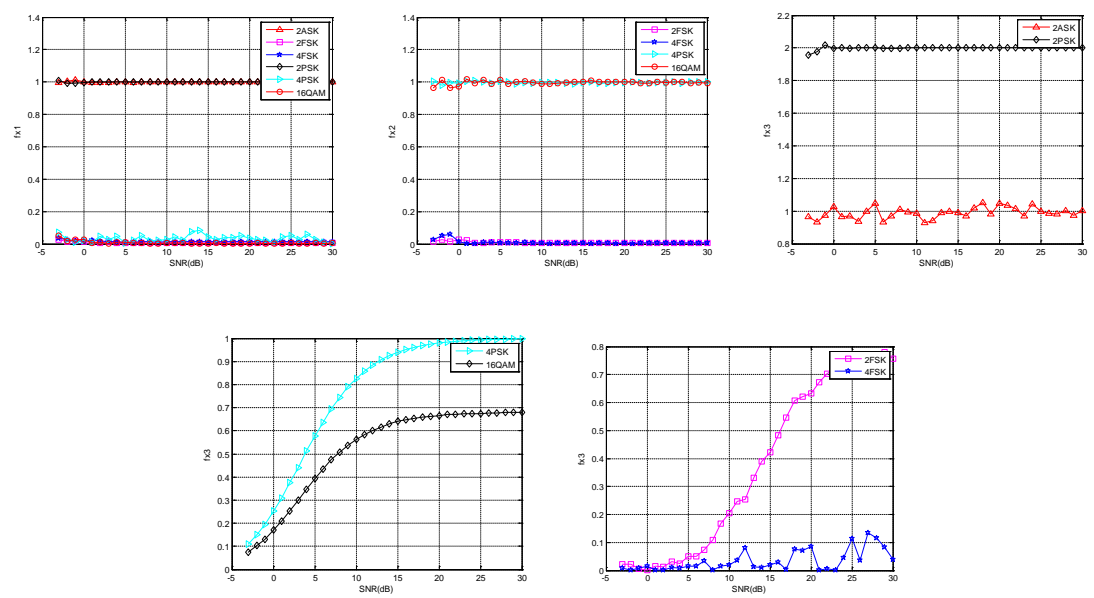

Figure 2-1 the simulation diagram of parameters one Figure 2-2 the simulation diagram of parameters two Figure 2-3 the simulation diagram of parameters three Figure 2-4 the simulation diagram of parameters three Figure 2-5 the simulation diagram of parameters three

\section{Acknowledgement}

This work was supported by the Fujian Natural Science Foundation of China (2013J01258), the key project in Fujian Province(2012H1012), the National Natural Science Foundation of China (61471309,61107023), Ph.D. Programs Foundation of Ministry of Education of China(20110121120020), supported by “ the Fundamental Research Funds for the Central Universities : 2013121023”

\section{Summary}

The automatic modulation identification for communication signals is important problem in the intercepted signal processing. This paper proposes a method that based on high order cumulants for identifying digital modulation signals, which can suppress the Gaussian white noise, and identify the six digital modulation signals. Theory analysis results prove the effectiveness of the algorithm.

\section{References}

[1] Orlic V, Dukic M L. Algorithm for automatic modulation classification in multipath channel based on sixth-order cumulants[C]//Telecommunication in Modern Satellite, Cable, and Broadcasting Services, 2009. TELSIKS'09. 9th International Conference on. IEEE, 2009: 423-426.

[2] A. Swami and B. M. Sadler, "Hierarchical digital modulation classification using cumulants," IEEE Trans. Commun., vol. 48, no . 3, pp. 416-429, Mar. 2000.

[3] Dobre O A, Bar-Ness Y, Su W. Higher-order cyclic cumulants for high order modulation classification[C]//Military Communications Conference, 2003. MILCOM'03. 2003 IEEE. IEEE, 2003, 1: 112-117.

[4] B. F. Beidas and C. L. Weber, "Higher-order correlation-based approach to modulation 0classification of digitally frequency-modulation signals,” IEEE J. Select. Areas Commun., vol. 13, pp. 89-101, Jan. 1995.

[5] O. A. Dobre, Y. Bar-Ness, and W. Su, "Higher-order cyclic cumulants for high order modulation classification,” in Proc. IEEE MILCOM, vol. 1, Oct. 2003, pp. 112-117.

[6] "Robust QAM modulation classification algorithm using cyclic cumulants," in Proc. IEEE Wireless Communications and Networking Conference, vol. 2, Mar. 2004, pp. 745-748.

[7] Sun H, Shen W, Wang Z, et al. Joint carrier frequency offset and impulse noise estimation for underwater acoustic OFDM with null subcarriers[C]//Oceans, 2012. IEEE, 2012: 1-4. 